\title{
Radiation, trapping and near-trapping in arrays of floating truncated cylinders
}

\author{
H.A. Wolgamot (hugh.wolgamot@eng.ox.ac.uk), R. Eatock Taylor \\ (r.eatocktaylor@eng.ox.ac.uk) and P.H. Taylor \\ (paul.taylor@eng.ox.ac.uk) \\ Department of Engineering Science, University of Oxford, Parks Road, Oxford, \\ OX1 3PJ, United Kingdom
}

\begin{abstract}
Circular arrays of fixed truncated vertical cylinders are known to support so called "sloshing near-trapped modes" when subjected to incident water waves of the appropriate frequency and direction. In this paper waves radiated from circular arrays of truncated cylinders oscillating independently in still water are considered. For a square array of four cylinders, peaks in the condition number of the radiation damping matrix are used to identify body motion modes of interest. Body motions which excite the same free surface motion local to the array as the sloshing neartrapped mode are associated with enhanced radiation from the array. In contrast, at lower frequencies oscillations with low wave radiation to infinity are found when the cylinders oscillate in phase, in either heave or surge. For an array with eight cylinders this almost wave-free behaviour is dramatically enhanced, and a very simple structure which appears to be "motion-trapping" may be constructed.
\end{abstract}

Keywords: Floating bodies, Trapped modes, Near trapping, Water waves

\section{Introduction}

In general, motion of rigid bodies floating in fluid with a free surface causes radiated waves to propagate along the free surface away from the body into the far field. In particular cases it is possible for bodies to oscillate at a specific frequency with no radiation. For a single body in 2- and 3-dimensions the phenomenon of wave-free heaving oscillation was investigated by Kyozuka and Yoshida [13]. The motivation in this case was the associated phenomenon of zero wave excitation force at the wave-free frequencies, as may be inferred from the Haskind relations [22]. Kyozuka and Yoshida created axisymmetric wave free bodies using singularities distributed along a vertical axis extending downwards from the free surface and the structures generated using this method had a maximum radius below the free surface. In the context of reducing the heave motions of a moored buoy the authors considered a mooring arrangement with linear spring stiffness such that the resonance frequency coincided with the zero-damping/zero-excitation frequency.

More recently the term motion-trapping structure has been introduced by McIver and McIver [19] to describe a structure which does

(C) 2013 Kluwer Academic Publishers. Printed in the Netherlands. 
not radiate at a particular frequency and, at that same frequency, satisfies a resonance condition in which the body inertia and fluid added mass terms are balanced by restoring force terms. The motiontrapping terminology is used because such a structure can evidently support a local bounded oscillation (at the appropriate frequency) in the vicinity of the oscillating structure with no radiation to infinity. Given an initial displacement from equilibrium, the motion of such a structure would eventually decay to undamped simple harmonic motion at the trapped mode frequency [26] (with the appropriate localised free surface displacement field close to the structure). The stiffness terms in the resonance condition could be hydrostatic and/or mooring terms and examples of each case have been studied. Once mooring terms are introduced, any wave-free structure can become a motion-trapping structure, so the more limited class of structure may be defined when only hydrostatic restoring forces are present. Motion-trapping structures without moooring forces were considered by McIver and McIver [19], who constructed 2-dimensional motion trapping structures, and in [20] extended this approach to 3-dimensions. Porter and Evans [26] started with a pair of rectangular cylinders or a thick-walled cylindrical shell (in 2- and 3-dimensions respectively) and varied the geometry to find motion trapping structures. Motion-trapping structures with mooring restraints were studied by Evans and Porter [5], who found that a moored submerged circular cylinder moving in heave or sway could be a motion trapping structure, and Newman [25] who analysed mooring stiffnesses on the continuum from $-\infty$ to $\infty$.

Motion trapping structures are so named to distinguish them from sloshing trapping structures, which held the original interest in trapping phenomena. Sloshing trapping structures are fixed structures which can support an oscillation of the free surface in the vicinity of the structure at a particular frequency, with no radiation to infinity. Sloshing trapping structures discovered include toroidal shapes derived using an indirect procedure, such as those found by McIver and McIver [18] and infinite arrays of bottom-mounted cylinders, as analysed by Evans and Porter [4] - or the equivalent case of a cylinder in a channel analysed by Callan et al [1]. In addition to these trapping structures, finite arrays of bottom mounted cylinders in linear [14] and circular [3] configurations were found to support near-trapped modes, where near trapping is characterised by amplifications in the exciting force on the whole array at a particular frequency. Near trapping was defined by Evans and Porter [3] as "a local oscillation in the vicinity of the array at a welldefined frequency which decays slowly as its energy leaks away due to wave radiation at large distances". 
A third class at the intersection of these two, described by Fitzgerald and McIver [7], corresponds to passive trapping structures, which are sloshing trapped modes which exert no force on the body, thereby allowing the body to be freely floating without any body motion or associated energy loss through radiation. These modes will not be considered here.

The motion trapping structures above have generally been in the context of single degree of freedom systems. When considering multiple modes, wave free oscillations of axisymmetric structures in surge and pitch may be simply constructed by selecting appropriate motion amplitudes and phases. Porter and Evans [27] found that pairs of floating 2-dimensional circular cylinders moving in heave and sway could act as a motion trapping structure at particular frequencies.

The existence of trapping structures is also related to questions of uniqueness of solutions to the governing equations in the cases of fixed structures (sloshing trapping) or free floating structures (motion trapping). Recent generalisations of the original work of John [8] by Kuznetsov and Motygin [12], [11] have extended the original single floating body theories to include bodies with multiple surface-piercing elements or submerged bodies.

In this paper we consider arrays of oscillating truncated cylinders; both sloshing and motion trapping (or near-trapping) effects are relevant for the array.

\section{Four cylinders}

\subsection{LINEAR THEORY}

Throughout this paper, and in the literature discussed above, linear potential flow analysis is used. Thus, the fluid motion is represented by a velocity potential $\phi$, with the sinusoidal time dependence $e^{-\mathrm{i} \omega t}$ removed, which satisfies

$$
\nabla^{2} \phi=0
$$

throughout the fluid and the condition

$$
\left[\frac{\partial \phi}{\partial z}\right]_{z=-h}=0
$$

on the seabed and

$$
g \frac{\partial \phi}{\partial z}-\omega^{2} \phi=0
$$

on the free surface.

Considering $N$ rigid bodies oscillating in the absence of incident waves, the total velocity potential may be decomposed into radiation 
potentials $\phi_{j}$ proportional to the body displacments $\xi_{j}$

$$
\phi=\operatorname{Re}\left\{\sum_{j=1}^{6 N}-\mathrm{i} \omega \hat{\xi}_{j} \phi_{j}\right\} .
$$

The radiation potentials may be determined using a further boundary condition on the body surface of a moving rigid body with normal $\boldsymbol{n}$

$$
\frac{\partial \phi_{j}}{\partial n}=n_{j} .
$$

Integrating fluid pressures over the body surface in the standard manner, the added mass and damping matrices may be expressed in terms of the radiation potentials

$$
-\mathrm{i} \omega \iint_{\mathrm{S}_{\mathrm{B}}} \frac{\partial \phi_{i}}{\partial n} \phi_{j} \mathrm{~d} \mathrm{~S}=B_{i j}-\mathrm{i} \omega A_{i j}
$$

where $B_{i j}$ and $A_{i j}$ are the $i, j$-elements of the added mass and damping matrices and $\mathrm{S}_{\mathrm{B}}$ is the submerged body surface. When the bodies may only move $M$ modes it is evident from (4) that only these modes need be considered in the damping matrix, which becomes $M \times M$.

\subsection{Four CYLINDERS}

A simple four cylinder array, shown in Figure 1, was considered by Siddorn and Eatock Taylor [28] in developing a semi-analytical method for scattering and radiation by arrays of truncated cylinders. The array had cylinders at the corners of a square, where the cylinders each had radius $a$, and draft $d=2 a$, the total fluid depth was $h=4 a$ and the square side length was $4 a$. This configuration had previously been investigated in the context of multiple scattering-type methods for analysing array forces and hydrodynamic coefficients by Kagemoto and Yue [9], who presented exciting force plots and by Mavrakos [16], who presented some plots of damping coefficients for the entire structure and individual elements of the damping matrix for independent motions.

Siddorn and Eatock Taylor found that their array could support a sloshing near-trapped mode at $k a=1.66$, which could be excited by waves incident on the fixed structure from $\beta=45^{\circ}$. Radiation damping coefficients were also presented and the condition number of the $(4 \times 4)$ heave, surge and $(8 \times 8)$ combined surge and sway damping matrices were presented to illustrate the radiation behaviour of the array. The condition number, $\kappa$, of a matrix $\boldsymbol{A}$ is defined as $\kappa(\boldsymbol{A})=\|\boldsymbol{A}\|\left\|\boldsymbol{A}^{-1}\right\|$, relative to a particular matrix norm [29],[10]; a singular matrix has 
infinite condition number and well conditioned matrices have low condition number. In each condition number plot in [28] two peaks were found in the range $0<k a<2$, which were assessed as being associated with near wave-free modes for the array. A connection was made with wave energy devices, and it was postulated that near wave-free modes may be associated with large absorption of wave energy.

Figures 2 and 3 display, respectively, the variation of condition number of the heave and surge damping matrices with frequency, including the original data of Siddorn and Eatock Taylor, and calculations performed with the boundary element code DIFFRACT. The condition number is calculated using the 1-norm (or equivalently, since the damping matrix is symmetric, the $\infty$-norm) as this permits direct comparison to Siddorn and Eatock Taylor. In Figure 4 the condition number of the radiation damping matrix is plotted against frequency, where the bodies may move only in a single horizontal mode at $45^{\circ}$ to the $x$-axis, which corresponds to surge along the $x^{\prime}$-axis displayed in Figure 1. In this paper surge will be understood to mean horizontal motion along the $\mathrm{x}$-axis and $x^{\prime}$-surge to mean horizontal motion in this alternative orientation. Figure 4 also displays the condition number for arbitrary horizontal oscillations (surge and sway) and the damping coefficients for both cases in this Figure are calculated using DIFFRACT. This code originated in the work of Chau [2] and has been extended and extensively validated over the ensuing years (e.g. [32]). This program can solve the diffraction and radiation problems for fixed and moving bodies at first and second-order (though only linear calculations are performed here). For linear calculations the submerged surfaces and inner water planes of the bodies are meshed with quadratic elements. Boundary element programs are now a common tool and have been used to analyse trapping and near-trapping structures, for example, by Newman [24]. In the present case DIFFRACT was validated against the published semi-analytical results of Siddorn and Eatock Taylor [28] and excellent agreement was found.

Also shown in Figures 2 and 3 for comparison is the condition number of the damping matrix when a small-body approximation is made, such that there is no scattering by the devices. These results, using the point absorber model common in wave power studies (e.g. [17]), show the same peaks, but indicate the effect of scattering, which is to shift the peaks in frequency. The elements of the damping matrix in this case are proportional to Bessel functions [17], such that in heave

$$
B_{r s}^{S B}=J_{0}\left(k d_{r s}\right)
$$

where $d_{r s}$ is the inter-device spacing while in surge

$$
B_{r s}^{S B}=J_{0}\left(k d_{r s}\right)-J_{2}\left(k d_{r s}\right) \cos \left(2 \alpha_{r s}\right)
$$


where $B_{r s}$ denotes the non-dimensional damping force on the $r^{t h}$ cylinder due to unit motion of the $s^{\text {th }}$ cylinder, with the other cylinders held fixed and $\alpha_{r s}$ is the direction of the $r$ th body from the $s$ th. The small-body approximation employed, denoted here by superscript $S B$, is not expected to be accurate over most of the range considered, but is of interest as a comparison when interference is present but scattering is neglected.

Peaks in the condition number of the heave damping matrix for the full problem occurred at $k a=0.67$ and $k a=1.64$, while the peaks for the surge damping matrix occurred at $k a=0.86$ and $k a=1.68$. The low-frequency peaks in Figure 4 occurred at $k a=0.90$ for $x^{\prime}$-surge and $k a=0.88$ for combined surge and sway while the frequencies of the second peak in each case matched that for surge in Figure 3. It is clear that there are two families of peak - in each case there is a low frequency peak at $k a<1$ and a second peak close to the sloshing near-trapping wavenumber. The body motions around each peak are of interest and in this section we seek to investigate these modes.

\subsection{BODY MODES}

As the damping matrix is symmetric, the condition number calculated using the 2-norm is the ratio of the largest to smallest eigenvalues of the damping matrix [29]. That is,

$$
\kappa_{2}(\boldsymbol{B})=\|\boldsymbol{B}\|_{2}\left\|\boldsymbol{B}^{-1}\right\|_{2}=\frac{\lambda_{\max }}{\lambda_{\min }} .
$$

Hence the peaks in the condition number correspond to a large ratio of these eigenvalues. Since the damping matrix is symmetric the eigenvalues and associated eigenvectors are real and because the damping matrix is positive definite (when not singular) these eigenvalues must be positive. Further, the eigenvectors may be chosen to be orthogonal and the basis of eigenvectors used to diagonalise the damping matrix. Considering the expression for time-average radiated power from the array [6]:

$$
P_{R}=\frac{1}{2} \omega^{2} \boldsymbol{\xi}^{*} \boldsymbol{B} \boldsymbol{\xi}
$$

a change of basis transformation may be used to express the radiated power in the form

$$
P_{R}=\frac{1}{2} \omega^{2}\left(\lambda_{1}\left|y_{1}\right|^{2}+\lambda_{2}\left|y_{2}\right|^{2}+\cdots+\lambda_{n}\left|y_{n}\right|^{2}\right)
$$

where $\boldsymbol{y}=\boldsymbol{X}^{T} \boldsymbol{\xi}$ is the vector of body displacements in the new basis and $\boldsymbol{X}$ is the change of basis matrix with columns equal to the 
normalised eigenvectors. Hence the eigenvalues are proportional to radiated power from the array (or, equivalently, power required to drive the array) in harmonic motion with unit amplitude in the displacements $y_{n}$, i.e. the eigenvectors in the original basis. The smallest eigenvalue at a given frequency must correspond to minimum radiation from the array (per unit amplitude displacement) and the largest eigenvalue maximum radiation. It is noted that in Figures 2 to 4 the condition number was evaluated using the 1-norm and the preceeding discussion is predicated on use of the 2-norm. However, the peaks occur at the same frequencies in most cases, an exception being for $x^{\prime}$-surge where there is a change of approximately $k a=0.01$ in the peak frequency.

\subsection{LOW FREQUENCY MODES}

Plotting the eigenvalues over the frequency range of interest should reveal which modes correpond to the peaks in condition number. In Figures 5 and 6 the eigenvalues for the heave and surge damping matrices are plotted against frequency. The eigenvectors in each case are those expected from symmetry considerations. Note that the extra symmetry in the heave case means that there is an eigenvalue of multiplicity 2, such that there is choice in the orthogonal eigenvectors.

The lowest-wavenumber peaks in the heave (along the vertical $z$ axis) and surge (along the $x$-axis) condition number plots were hence found to give mode shapes with all bodies oscillating in phase, i.e.:

- Heave: $k a=0.67$ corresponds to $\boldsymbol{\xi}_{z}=[1,1,1,1]$

- Surge: $k a=0.86$ corresponds to $\boldsymbol{\xi}_{x}=[1,1,1,1]$

Thus, the first peaks in each case correspond to a minimum of radiation from an oscillation of all cylinders moving as a single rigid body. Damping coefficients for the cylinder array moving as a single body were calculated using DIFFRACT, and are shown in Figures 8 and 9, where a minimum may be observed at the expected frequency in each case. In these Figures the damping of the multi-column structure is compared to four times the damping of a single cylinder in the same mode. The minimum in the surge damping coefficient was presented for this structure in the results of Kagemoto and Yue [9] and Mavrakos [16], although no comment was made on the results. Similarly the minimum in the heave damping for four truncated cylinders has been presented previously for slightly different geometries, for example by Yilmaz [31]. The minima in damping are quite significant, with a reduction in damping from the reference case of four isolated cylinders by a factor of 20 or 30 . In the heave case the minimum is more pronounced - logarithmic 
axes are used to make this clear. As identified by Siddorn and Eatock Taylor, these motions are near wave-free modes.

For comparison, the same analysis was performed for the lowest frequency peaks in the heave and surge condition number plots for the small body approximation. In each case the modes identified were those listed above for the case with scattering included.

The damping coefficient for the four cylinders heaving in phase may be expressed as a combination of the elements of the heave damping matrix for the array, according to:

$$
B_{z}=4\left(B_{11}+2 B_{21}+B_{41}\right)
$$

using the symmetry of the problem. ( $B_{r s}$ denotes the heave damping coefficient for the bodies moving independently as in Equations (7) and (8) and $B_{z}$ has been used to identify the damping of the cylinders heaving as a single structure.) This provides some insight into what is occurring at the near-wave-free frequency. In a small body approximation, terms $B_{r s}$ are given by Equation (7). Therefore, under this theory, $B_{21}$ and $B_{41}$ will become negative when the 2-1 and 4-1 separations $4 a$ and $4 \sqrt{2} a$ cause the argument to exceed 2.4048, the first zero of the Bessel function. The $B_{41}$ term will become negative first, followed by the $B_{21}$ term. At some middle point corresponding to optimum cancellation of the radiated waves from the respective cylinders the overall heave damping will reach its minimum. This behaviour of the individual coefficients is shown in Figure 10. The effect of scattering shifts these behaviours in frequency, as shown in Figure 11, and in particular the effect of $B_{21}$ is lessened relative to $B_{41}$ so that when scattering is included the minimum shifts to lower frequency.

For $x^{\prime}$-surge the eigenvalues are different and two of them change over the frequency range. The eigenvalues in this case are plotted in Figure 7.

When $x^{\prime}$-surge is considered, the smallest eigenvalue is no longer associated with a rigid body mode. While it is clear from symmetry considerations that the rigid body mode at $45^{\circ}$ must also have low damping, it may not represent the best approximation of a wave-free mode. The plots of condition number in Figures 3 and 4 support this conclusion, as the condition number for $x^{\prime}$-surge is higher than for the surge case. In fact, the smallest eigenvalue is associated with a mode of the form $x=[1, \gamma, \gamma, 1]$, as might be expected from symmetry. At the near wave-free frequency the free parameter $\gamma$ was found to be $\gamma=1.19$. 


\subsubsection{Free surface motions}

Having identified the body motion modes for the first condition number peaks, it is of interest to consider the corresponding motions of the free surface. The free surface amplitudes for unit excursion amplitude in sinusiodal heave oscillations are shown in Figure 12, for the near wavefree frequency $k a=0.67$ (Figure $12(\mathrm{c})$ ) and for frequencies above (e) and below (a) this value. Figures 12 (b), (d) and (f) show the free surface amplitudes with the scale limited to emphasise the radiation pattern outside the array.

It is readily apparent that there is much less radiation outside the array at the near wave-free frequency than at adjacent frequencies, as expected. The radiation patterns produced are also interesting. Clearly in all cases the structure internal to the array is a simple monopole pumping mode. Of more interest is the behaviour external to the array. At frequencies lower than the near wave-free frequency, radiation is dominated by beams along the directions of the diagonals of the array (the 4-1 direction), while at higher frequencies, radiation is dominated by beams perpendicular to the faces of the square. At the near wave-free frequency, it may be seen that an optimum intermediate between these two behaviours is reached, and elements of both patterns are present in the much lower total radiation.

Similar behaviour may be observed in Figure 13, which displays the free surface around the rigid body array moving in surge along the $x$ axis. In this case the mode is no longer a monopole, with a nodal line clearly observable down the plane of symmetry of the array. The free surface is $\pi$ out of phase on either side of this line.

\subsection{Higher FREQUenCy MOdeS}

In addition to the major peaks analysed in Section 2.4, the condition number plots shown in Figures 2, 3 and 4 each contain a peak at a frequency close to the sloshing near-trapping wavenumber for the array, identified by Siddorn and Eatock Taylor [28] as $k a=1.66$. It may be seen in Figures 6 and 7 that there is no small eigenvalue associated with the frequency of peak condition number in surge. However, there is one mode which has a substantially higher eigenvalue than the others, leading to the elevated condition number - similar behaviour is apparent if the scale on the heave plot is adjusted. The modes (eigenvectors) corresponding to the peak eigenvalues were found to be:

- Heave: $k a=1.64$ corresponds to $\boldsymbol{\xi}_{z}=[-1,1,1,-1]$

- Surge: $k a=1.68$ corresponds to $\boldsymbol{\xi}_{x}=[1,-1,1,-1]$

- $\quad x^{\prime}$-surge: $k a=1.68$ corresponds to $\boldsymbol{\xi}=[1,0,0,-1]$ 
The plot of the eigenvalues given for the heave case given in Figure 5 may be made more useful by normalising by the damping for an isolated cylinder, as shown in Figures 14. Evidently the ratio of the damping coefficients is equivalent to the ratio of the radiated power from the array with unit amplitude to the radiated power from the cylinder with unit amplitude. In this figure it may be clearly seen that the mode listed above corresponds to maximal radiation from the array (at the appropriate frequency), in contrast to the minimum power radiated by the near wave-free modes occurring at lower frequency which were analysed in Section 2.4. (It may be noted in this figure that in the zerofrequency limit modes with zero net displacement appear to asymptote to zero radiated power, while modes with a net displacement of four times a single cylinder approach a limit of four, as expected.)

As already identified, the frequencies of these modes are close to the sloshing near-trapping frequency for the fixed structure. This sloshing near-trapped mode has been analysed previously by Evans and Porter [4], where the layout of the cylinders in the horizontal plane was identical to the current case, but the cylinders extended throughout the water depth. The sloshing near-trapped mode manifests itself in the exciting forces on the fixed structure when waves of the appropriate frequency are incident along the $x^{\prime}$-axis (i.e. from $45^{\circ}$ ). As the incident wave direction is varied away from this value the extent to which this mode is excited gradually decreases. Nevertheless, according to the Haskind relations (e.g. [22]), the effect of the sloshing near-trapped mode is present in the damping coefficients for the moving structure as a result of the integrated effect over all incident wave angles (in this case 0 to $45^{\circ}$ ). Individual elements of the heave damping matrix have been presented in Figure 11. Elements of the $x^{\prime}$-surge damping matrix are shown in Figure 15, and in both figures the effect of the sloshing neartrapped mode around $k a=1.66$ is clear. This behaviour is similar to that reported by Newman [24], who investigated hydrodynamic coefficients for a true sloshing trapping structure (a toroidal body) and found that singular behaviour was observed at the trapping frequency. In the case of near-trapping magnification of the damping coefficients may be expected.

Evans and Porter investigated the geometric pattern of the sloshing near-trapped mode. Following this approach, the scattered free surface excited by an incident wave of the appropriate frequency, with the incident wave then removed, is shown in Figure 16 (as shown in [4]), as calculated by DIFFRACT. It is apparent that peaks in the free surface amplitudes are associated with each cylinder in a diagonal pattern, with opposite diagonals out of phase. Evans and Porter [4] also determined 
the pattern of the (horizontal) forces on the array due to this neartrapped mode - this is shown in Figure 17.

It is apparent that the phase difference of $\pi$ between the force on cylinders 1 and 4 shown in Figure 17 is reproduced in the surge motion mode $\left[\begin{array}{llll}1 & 0 & 0 & -1\end{array}\right]$ discussed above for $x^{\prime}$-surge oscillations of the four cylinders. This diagonal structure in the mode of oscillation arises from the effects on the elements of the damping matrix - in surge only the 1-1 and 4-1 coefficients are affected, as clearly shown in Figure 15. Diagonal structure is also apparent in Figure 18, which shows the free surface around the array when oscillating in $x^{\prime}$ surge mode $\boldsymbol{x}=\left[\begin{array}{llll}1 & 0 & 0 & -1\end{array}\right]$ and clearly resembles that in Figure 16.

It thus appears that the higher-frequency peak in the condition number curves is caused by magnification of the elements of the damping matrix associated with the sloshing near-trapped mode at that wavenumber for the array. Even for the surge $\left(0^{\circ}\right)$ case, which is less conducive to exciting the diagonal structure of the near-trapped mode, the mode [ $\left.\begin{array}{llll}1 & -1 & 1 & -1\end{array}\right]$ with the best approximation of diagonal structure is found. The mode shapes identified and considered in this section appear to be the opposite of wave-free, instead corresponding to maximal radiation from the array.

The point absorber condition number curves also shown in Figures 2 to 4 have peaks at similar frequencies to the higher peaks in the DIFFRACT analysis. When the eigenvalue analysis is applied to the small body damping matrix for heave, the $\left[\begin{array}{lll}1 & -1 & -1\end{array}\right]$ mode is obtained, but in this case with the eigenvalue changing sign in the expected manner. This mode is the same as the mode considered above, with full diffraction included, but in this case may be shown to correspond to a minimum in radiated power - i.e. a near wave-free mode. Similarly, for surge, the small body approximation theory peak is associated with a mode [1 - $0.64-0.641]$ which is also near wave-free. The small body theory is well outside the range in which it could be expected to give accurate results, but these results are interesting as they indicate that without scattering there may still be interactions at similar frequencies, but the effect of the sloshing near-trapped mode changes the behaviour from cancellation of the far-field radiated wave to reinforcement.

When each cylinder is allowed to surge and sway, the condition number of the damping matrix at the sloshing near-trapping frequency is substantially increased, as may be seen in Figure 4. In this case one mode is clearly identifiable as having the largest eigenvalue. If the $x^{\prime}-y^{\prime}$-axes are used, this is a combination of two perpendicular versions of the $x^{\prime}$-surge mode considered above, i.e. $\boldsymbol{\xi}=\left[\begin{array}{llllllll}1 & 0 & 0 & 1 & 0 & -1 & -1 & 0\end{array}\right]$, where the modes are labelled [cylinder $1 x^{\prime}$ surge, cylinder $1 y^{\prime}$ sway, cylinder 2 $x^{\prime}$ surge, ... . As is evident, this matches the pattern of forces depicted 
in Figure 17 for the force on a fixed array due to the sloshing neartrapped mode. This mode excites the sloshing near-trapping resonance more effectively than the $x^{\prime}$ surge mode alone, and is associated with a larger condition number and larger radiation from the array.

\section{8 cylinders}

The four-cylinder array considered previously might be regarded as a first approximation to an axisymmetric shape. Axisymmetric shapes with an enclosed free surface, or moonpool, are well known for supporting trapped modes (of sloshing, motion or passive-trapped type). In fact, resonances in the moonpool of 2-dimensional structures and rectangular and axisymmetric structures in 3-dimensions have been extensively studied independently of motion trapping, for example, by Yeung and Seah [30], Molin [21] and Mavrakos [15] (rectangular crosssection) or Newman [23] (circular cross-section), respectively. A series of resonances occur, with the lowest frequency resonance associated with a pumping or piston mode, in which the free surface elevation in the moonpool is non-zero, and a series of higher order resonances (sometimes termed sloshing modes, though this terminology will be avoided here). When the hydrodynamic coefficients are considered, these resonances are associated with zero damping and regions of negative added mass, though there is a distinction between rather smooth behaviour of the coefficients observed in the vicinity of the pumping mode and the much more abrupt changes in the vicinity of the higher order resonances. Porter and Evans [26] constructed motion trapping structures made of heaving axisymmetric shells of rectangular cross section by ensuring that the inertia terms in the frequency-domain equation of motion were balanced by hydrostatic stiffness at the same frequency as the zero-damping occurring around higher order moonpool resonances.

The four cylinders considered above behaved as a near-wave-free structure when heaving as a single rigid body at one frequency and could therefore, with the addition of a spring of the appropriate stiffness, become what might be termed a near motion-trapping structure. Calculations performed with three cylinders at the vertices of an equilateral triangle showed that a much weaker minimum in the heave damping coefficient was present in this case. It is of interest to consider the case with more cylinders, providing a better approximation of axisymmetric geometry. In this section we consider 8 cylinders laid out around the circumference of a circle, heaving as a rigid body; multiple-body modes will not be considered. 
The layout considered first was composed of 8 cylinders identical to those in Section 2, laid out with centres equally spaced around the circumference of a circle of radius $5 a$, with the water depth also unchanged at $4 a$. The heave damping coefficient for this structure is plotted against wavenumber in Figures 19 and (on logarithmic axes) 20.

It is apparent that there is strong wave-free behaviour at a wavenumber of approximately $k a=0.42$. In fact, the damping coefficients calculated by DIFFRACT at frequencies around the minimum (more accurately located at $k a=0.4216$ ) are very small negative numbers, though monotonically increasing toward zero with increasing mesh refinement. (Such very small negative damping at a wave-free frequency appears also to have been obtained by Newman [25], using WAMIT. These results would appear to be an artefact of the boundary element method in both codes.) The free surface around the heaving structure at this frequency is shown in Figure 21 and it is apparent that this mode is a piston mode similar to that obtained in the four cylinder case in Section 2.4.

The resonance condition necessary for motion trapping of a freely floating structure (in addition to zero damping) is

$$
-\omega^{2}(M+A(\omega))+C=0
$$

where $M$ is the mass of the floating body, $A(\omega)$ the frequency-dependent added mass and $C$ the hydrostatic stiffness. Equation 13 is not satisfied by the geometry considered, but the frequency at which it is can be adjusted by changing the draft of the cylinders. If the draft is increased by $3.4 \%$ - a perturbation selected using a trial-and-error procedure - the minimum damping frequency is reduced slightly to $k a=0.4205$. At this frequency, the value of the left hand side of Equation 13, normalised by $C$, is $3.4 \times 10^{-5}$, where DIFFRACT parameters are obtained from the finest mesh resolution used, with 1644 elements per cylinder and the default DIFFRACT value $g=9.807 \mathrm{~ms}^{-2}$ has been used. To the accuracy of the method used, the structure with draft $2.068 a$ appears to be a motion-trapping structure (which possesses neither true axisymmetry nor a moonpool), and can be constructed quite simply using this approach.

As reported above for the 8 cylinder geometry with unmodified draft, the damping values calculated by DIFFRACT for the case of increased draft around the minimum damping frequency were very small negative numbers. Negative damping values are unphysical, and it is therefore of interest to examine this behaviour in the context of mesh convergence. Figure 22 shows the heave damping coefficient for the structure with the increased draft around the minimum damping frequency, as calculated 
using 3 different meshes. For all 3 meshes, the minimum damping value is negative. Simple Richardson-type extrapolation was performed for all 11 frequencies individually and these values then interpolated in frequency using cubic splines, as shown in the dashed line in the Figure. The extrapolated (normalised) value of the damping at $k a=0.4205$ was calculated to be $5 \times 10^{-9}$, while the minimum damping using the spline interpolation occurred at $k a=0.420462$ and the corresponding damping was $1.2 \times 10^{-9}$. The significance of these values is in the convergence of the damping to a small positive number and the order of magnitude estimate of this value.

Another way to calculate the damping around the minimum damping frequency is to use reciprocity relationships between the excitation force acting on the body and the radiation damping, for example [22]:

$$
B_{i i}=\frac{k}{16 \pi J} \int_{0}^{2 \pi}\left|F_{i}(\beta)\right|^{2} \mathrm{~d} \beta .
$$

where $J$ is the power transported by a wave of wavenumber $k$. To this end, the exciting force at $k a=0.4205$ was evaluated for the three meshes considered above, for incoming wave directions in 2.5 degree increments between 0 and 22.5 degrees (due to symmetry of the structure this is all that is required). The real and imaginary parts of the exciting force at $k a=0.4205$ are shown in Figure 23. It may be seen that the exciting force is small but non-zero, and that the real part of the exciting force is a strong function of incoming wave direction, though the imaginary part is not. An extrapolation similar to that performed for the damping coefficients above was performed for each incoming wave angle, with the results plotted in Figure 23. Using Equation 14 to calculate the damping at this frequency yields a value of $6 \times 10^{-9}$ which agrees well with the value for this frequency estimated above. It is interesting to note the variation in exciting force with incoming wave angle - a true motion trapping structure, by virtue of the Haskind relations, would have zero exciting force at all incoming wave angles. This implies that a non-axisymmetric motion-trapping structure would have to have the same zero exciting force when the incoming wave was effectively encountering different geometries.

Porter and Evans [26] emphasised the difficulty of finding a motiontrapping frequency due to the blow-up of the hydrodynamic coefficients at frequencies surrounding the wave-free frequency. This phenomenon was not encountered here, probably because their motion trapping was associated with higher modes than the pumping mode considered here. The heave added mass coefficient is shown in Figure 24 and it may be seen that the behaviour is smooth. For structures with a moonpool, the piston mode is generally associated with smooth variation through 
a region of negative added mass (e.g. [30]), but negative added mass is not observed for this structure.

Higher resonances in the 8 cylinder problem are not associated with wave-free behaviour, as is apparent in Figure 20 where the small feature at $k a=1.02$ corresponds to an approximate higher resonance. The free surface around the structure oscillating in heave at this frequency is shown in Figure 25 and confirms that this is the first of the higher modes, mentioned, for example, by Yeung and Seah [30] in a 2 -dimensional context as being standing wave patterns. One can speculate that at this higher frequency, the 8 cylinders no longer approximate a ring well enough to give wave-free behaviour (or a close approximation to it). A contributing factor may also be that antinodes are located at the ring edge for this higher mode, which may be less well supported by the discrete cylinders. Even were wave-free behaviour observed at the higher mode, it is apparent that the resonance condition would not be satisfied.

In the context of sloshing near-trapping Evans and Porter [4] found that breaking the symmetry of the array by even a small perturbation could greatly reduce the magnitude of the near-trapping effect. To investigate whether a similar result holds in this case the damping coefficient for the 8 cylinder structure with modified draft was calculated with one cylinder displaced a distance of $0.1 a$ radially towards the centre of the array. It was found that the minimum damping coefficient increased by more than two orders of magnitude (and was shifted slightly in frequency), indicating that symmetry is of considerable importance. However, the damping of this distorted eight cylinder structure was still considerably lower than the four cylinder case considered earlier.

\section{Conclusions}

Aspects of wave radiation from arrays of floating truncated cylinders have been considered. A pumping mode with all cylinders heaving as a single rigid body appears to be a common feature of cylinders arranged in a circular fashion, and with sufficiently many cylinders approaches a wave-free mode. These wave-free modes may be simply adapted to form near motion-trapping structures by varying the cylinder geometry. As the pumping mode is not associated with rapid variation of the hydrodynamic coefficients this is relatively straightforward. It is remarkable that such a close approximation to a motion-trapping structure can be formed so simply, by a structure which has neither a true moonpool nor a complicated profile below the waterline. 
The elements of the damping matrix for such an array of truncated cylinders are affected when sloshing near-trapping modes are present. Such features may give rise to peaks in the condition number similar to near wave-free modes, but do not necessarily correspond to such phenomena; indeed, radiation in the appropriate modes at such frequencies appears to be maximised.

The rigid body pumping modes may be of interest for TLPs or semisubmersible platforms - structures with arrays of cylinders constrained to move together. Multiple-body modes at sloshing near-trapping frequencies might possibly be useful in a wave energy context, where the efficient, directional radiation of these modes may be desirable. However, the high frequency and low bandwidth of these modes could negate such advantages.

\section{Acknowledgements}

H. Wolgamot was supported by a University of Oxford Clarendon Fund Scholarship.

\section{References}

1. Callan, M., C. Linton, and D. Evans: 1991, 'Trapped modes in two-dimensional waveguides'. Journal of Fluid Mechanics 229, 51-64.

2. Chau, F.: 1989, 'The second order velocity potential for diffraction of waves by fixed offshore structures'. Ph.D. thesis, University College London.

3. Evans, D. and R. Porter: 1997, 'Near-trapping of waves by circular arrays of vertical cylinders'. Applied Ocean Research 19, 83-99.

4. Evans, D. and R. Porter: 1999, 'Trapping and near-trapping by arrays of cylinders in waves'. Journal of Engineering Mathematics 35(1), 149-179.

5. Evans, D. and R. Porter: 2007, 'Wave-free motions of isolated bodies and the existence of motion-trapped modes'. Journal of Fluid Mechanics 584(1), 225234.

6. Falnes, J.: 2002, Ocean waves and oscillating systems. Cambridge University Press.

7. Fitzgerald, C. and P. McIver: 2010, 'Passive trapped modes in the water-wave problem for a floating structure'. Journal of Fluid Mechanics 657, 456-477.

8. John, F.: 1950, 'On the motion of floating bodies II. Simple harmonic motions'. Communications on pure and applied mathematics 3(1), 45-101.

9. Kagemoto, H. and D. Yue: 1986, 'Interactions among multiple threedimensional bodies in water waves: an exact algebraic method'. Journal of Fluid Mechanics 166, 189-209.

10. Kreyszig, E.: 2006, Advanced engineering mathematics. John Wiley \& Sons.

11. Kuznetsov, N. and O. Motygin: 2011, 'On the coupled time-harmonic motion of water and a body freely floating in it'. Journal of Fluid Mechanics 679(1), $616-627$. 
12. Kuznetsov, N. and O. Motygin: 2012, 'On the coupled time-harmonic motion of deep water and a freely floating body: trapped modes and uniqueness theorems'. Journal of Fluid Mechanics 703, 142.

13. Kyozuka, Y. and K. Yoshida: 1981, 'On wave-free floating-body forms in heaving oscillation'. Applied Ocean Research 3(4), 183-194.

14. Maniar, H. and J. Newman: 1997, 'Wave diffraction by a long array of cylinders'. Journal of Fluid Mechanics 339, 309-330.

15. Mavrakos, S.: 1988, 'Hydrodynamic coefficients for a thick-walled bottomless cylindrical body floating in water of finite depth'. Ocean Engineering 15(3), 213-229.

16. Mavrakos, S.: 1991, 'Hydrodynamic coefficients for groups of interacting vertical axisymmetric bodies'. Ocean Engineering 18(5), 485-515.

17. McIver, P.: 1994, 'Some hydrodynamic aspects of arrays of wave energy devices'. Applied Ocean Research 19, 283-291.

18. McIver, P. and M. McIver: 1997, 'Trapped modes in an axisymmetric waterwave problem'. The Quarterly Journal of Mechanics and Applied Mathematics 50(2), 165-178.

19. McIver, P. and M. McIver: 2006, 'Trapped modes in the water-wave problem for a freely floating structure'. Journal of Fluid Mechanics 558(1), 53-67.

20. McIver, P. and M. McIver: 2007, 'Motion trapping structures in the threedimensional water-wave problem'. Journal of Engineering Mathematics 58(1), $67-75$.

21. Molin, B.: 2001, 'On the piston and sloshing modes in moonpools'. Journal of Fluid Mechanics 430(1), 27-50.

22. Newman, J.: 1976, 'The interaction of stationary vessels with regular waves'. In: Proceedings of the 11th Symposium on Naval Hydrodynamics. pp. 491-501.

23. Newman, J.: 1977, 'The motions of a floating slender torus'. Journal of Fluid Mechanics 83, 721-735.

24. Newman, J.: 1999, 'Radiation and diffraction analysis of the McIver toroid'. Journal of Engineering Mathematics 35(1), 135-147.

25. Newman, J.: 2008, 'Trapping of water waves by moored bodies'. Journal of Engineering Mathematics 62(4), 303-314.

26. Porter, R. and D. Evans: 2008, 'Examples of trapped modes in the presence of freely floating structures'. Journal of Fluid Mechanics 606, 189-208.

27. Porter, R. and D. Evans: 2009, 'Water-wave trapping by floating circular cylinders'. Journal of Fluid Mechanics 633, 311.

28. Siddorn, P. and R. Eatock Taylor: 2008, 'Diffraction and independent radiation by an array of floating cylinders'. Ocean Engineering 35, 1289-1303.

29. Trefethen, L. N. and D. Bau III: 1997, Numerical linear algebra, No. 50. Society for Industrial and Applied Mathematics.

30. Yeung, R. and R. Seah: 2007, 'On Helmholtz and higher-order resonance of twin floating bodies'. Journal of Engineering Mathematics 58(1), 251-265.

31. Yilmaz, O.: 1998, 'Hydrodynamic interactions of waves with group of truncated vertical cylinders'. Journal of Waterway, Port, Coastal and Ocean Engineering 124(5), 272-279.

32. Zang, J., R. Eatock Taylor, and P. Taylor: 2005, 'Second-order wave forces and free-surface elevation around a moored ship in steep uni-directional and spread waves'. In: Proceedings of the 4 th International Conference on Marine Computational Fluid Dynamics, Southampton, UK. pp. 53-58. 


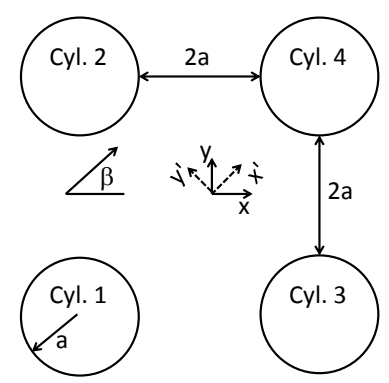

Figure 1. Layout of four cylinder array analysed by Siddorn and Eatock Taylor.

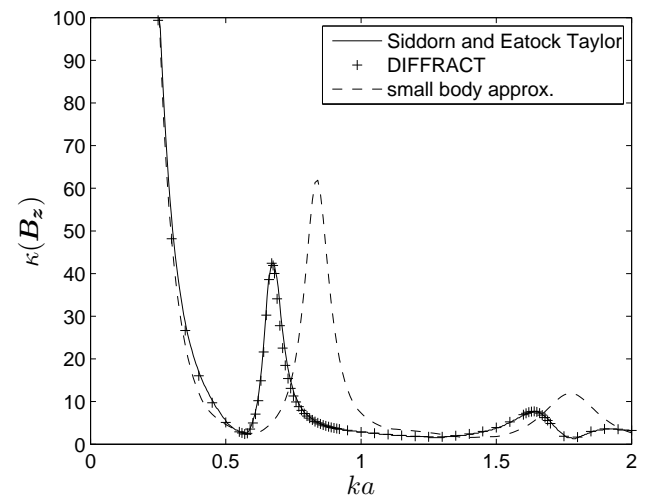

Figure 2. Condition number of heave damping matrix.

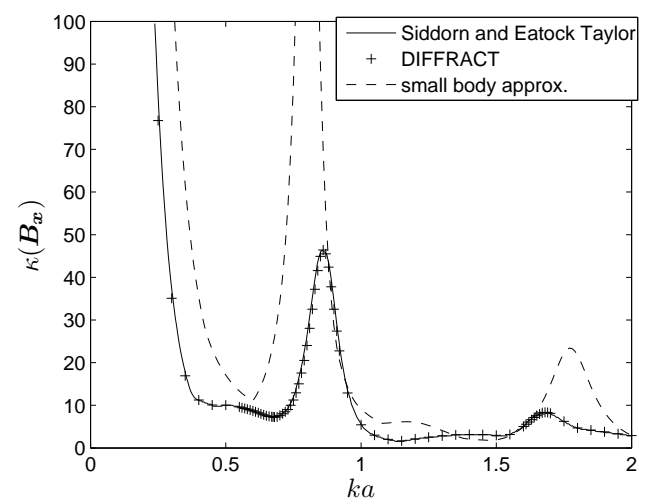

Figure 3. Condition number of surge damping matrix (surge along the $x$-axis). 


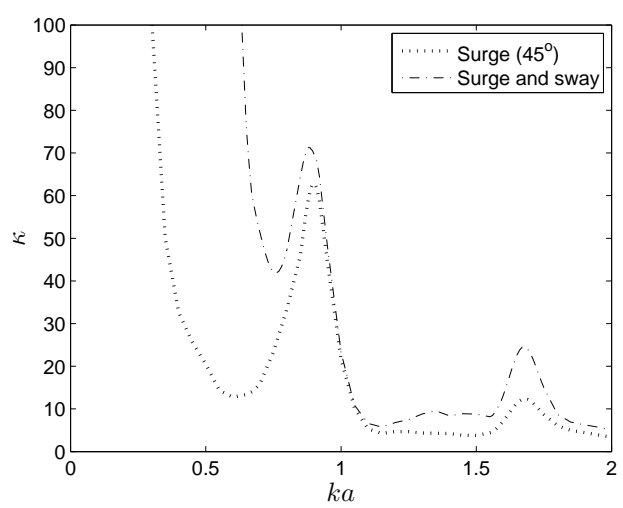

Figure 4. Condition number of $x^{\prime}$-surge damping matrix and surge-sway damping matrix calculated using DIFFRACT.

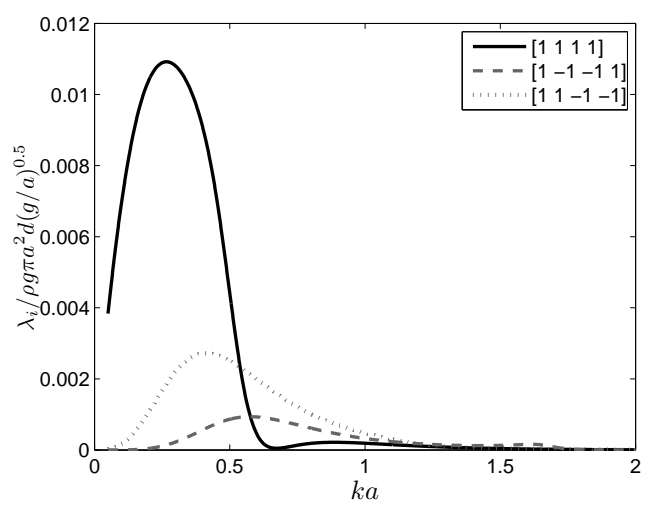

Figure 5. Eigenvalues of damping matrix (heave). The eigenvalue associated with the $\left[\begin{array}{llll}1 & 1 & -1 & -1\end{array}\right]$ eigenvector is of multiplicity 2 .

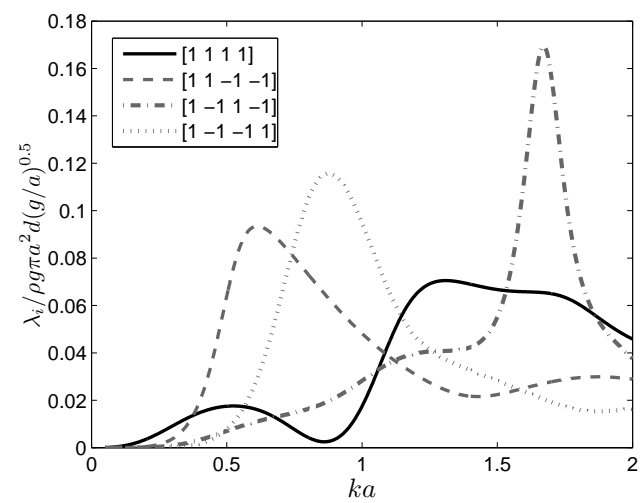

Figure 6. Eigenvalues of damping matrix (surge). 


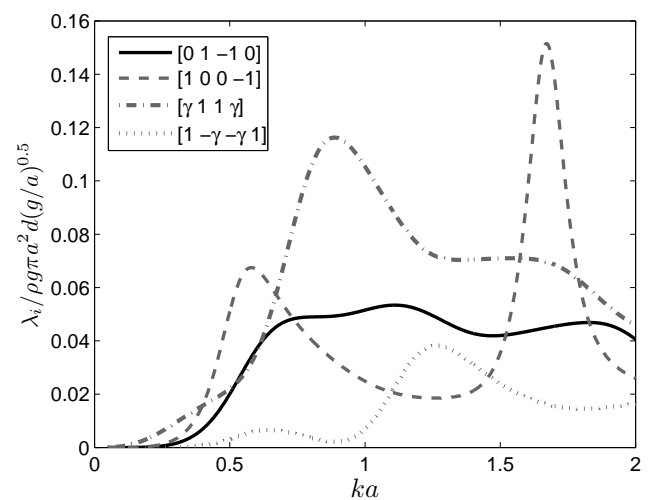

Figure \%. Eigenvalues of damping matrix (surge).

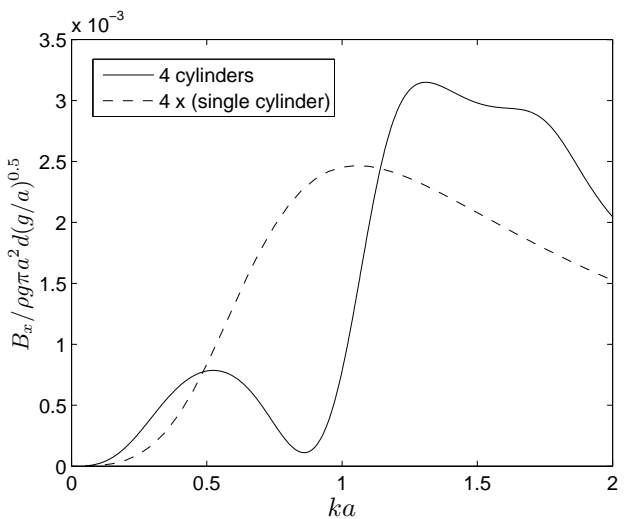

Figure 8. Surge damping for four cylinders moving as single structure. 


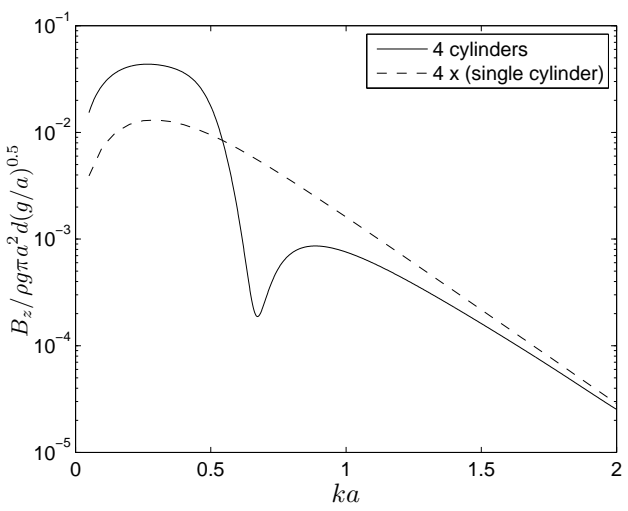

Figure 9. Heave damping for four cylinders moving as single structure.

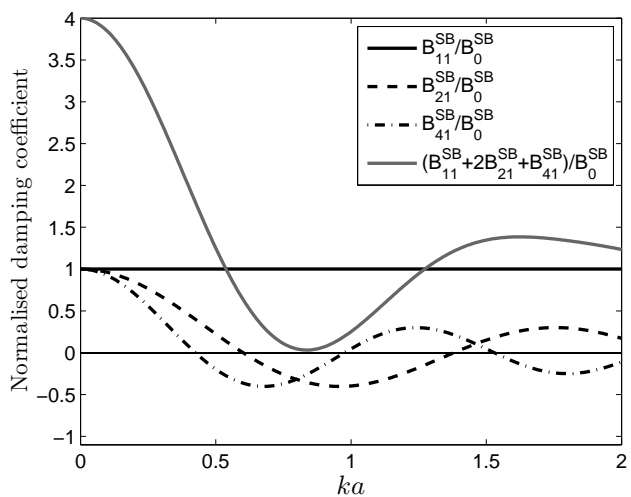

Figure 10. Elements of the damping matrix for four cylinders in heave, calculated using the small body approximation. 


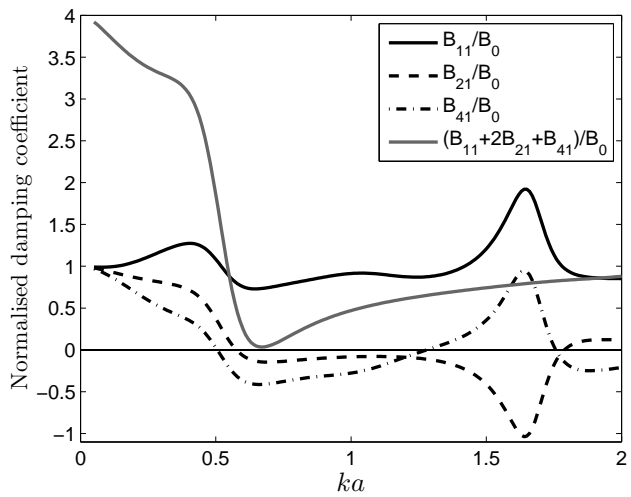

Figure 11. Elements of the damping matrix for four cylinders in heave, calculated using DIFFRACT. 


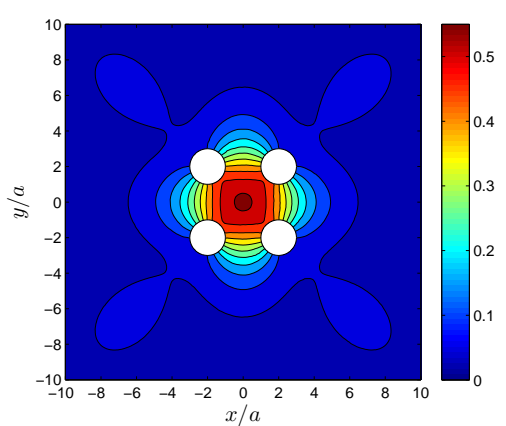

(a) $k a=0.60$

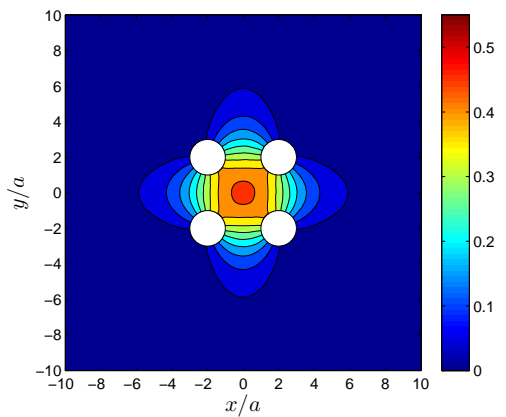

(c) $k a=0.67$

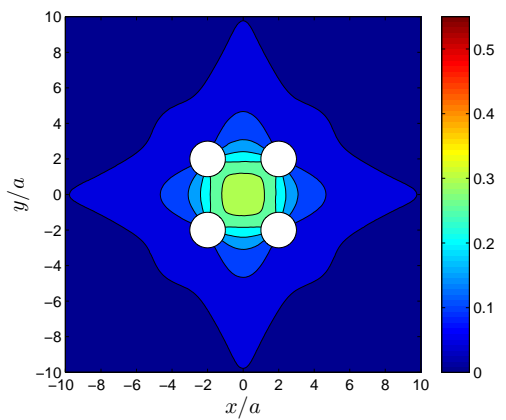

(e) $k a=0.80$

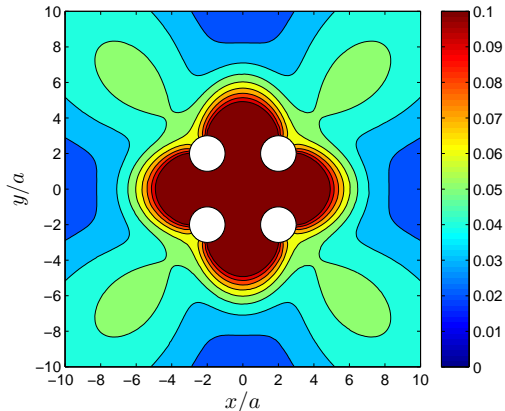

(b) $k a=0.60$

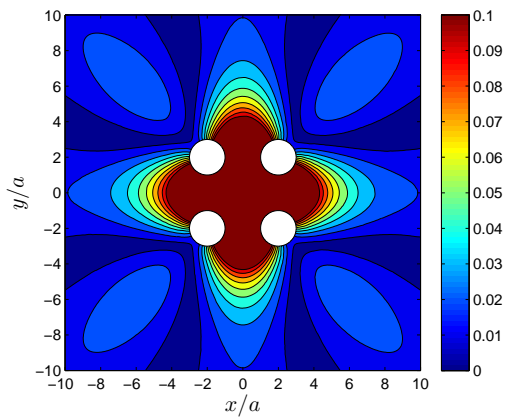

(d) $k a=0.67$

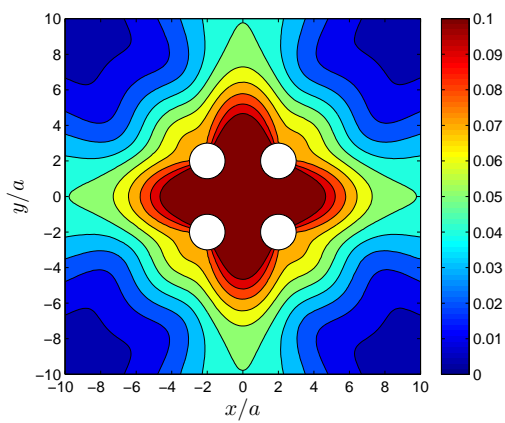

(f) $k a=0.80$

Figure 12. Free surface amplitude moduli, for heave radiation in rigid-body mode with unit amplitude at minimum damping frequency $k a=0.67$ and adjacent frequencies. Figures (b), (d) and (f) are shown with a limited amplitude scale so that the structure external to the array may be seen. 


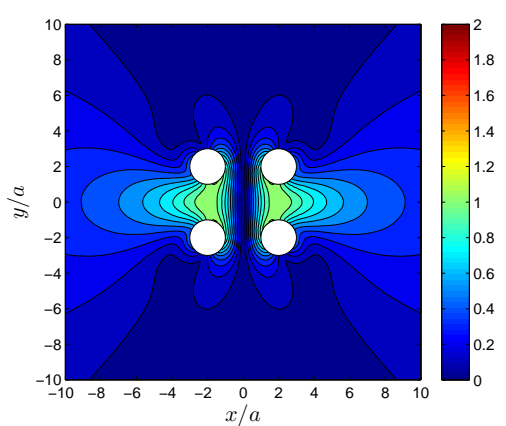

(a) $k a=0.75$

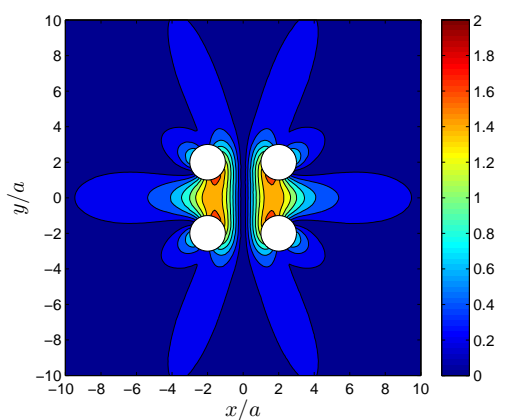

(c) $k a=0.86$

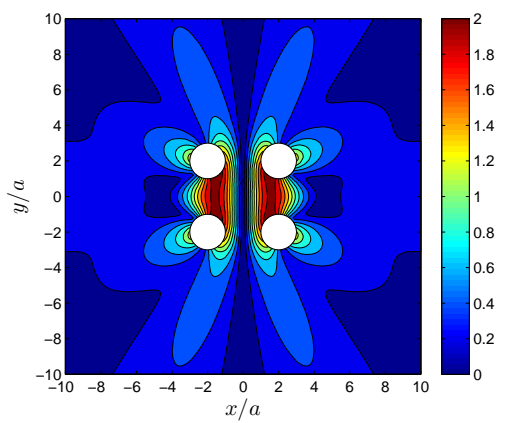

(e) $k a=0.95$

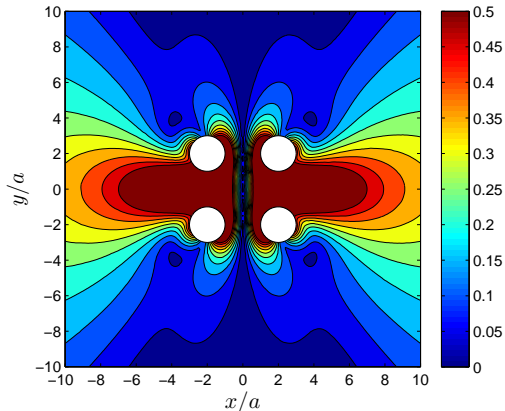

(b) $k a=0.75$

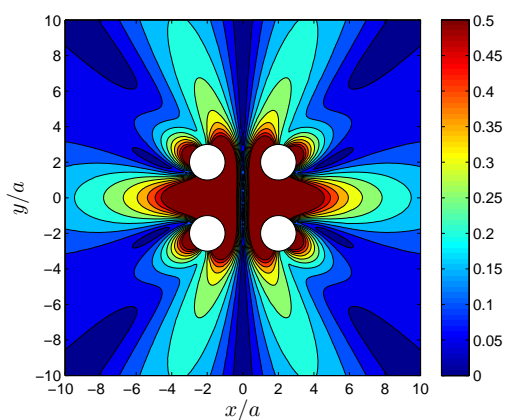

(d) $k a=0.86$

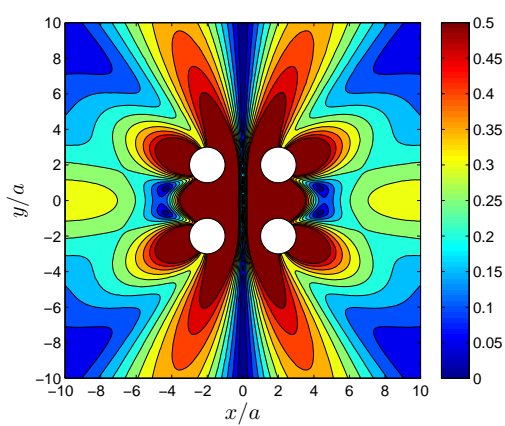

(f) $k a=0.95$

Figure 13. Free surface amplitude moduli, for surge radiation in rigid-body mode with unit amplitude at minimum damping frequency $k a=0.86$ and adjacent frequencies. Figures (b), (d) and (f) are shown with a limited amplitude scale so that the structure external to the array may be seen. 


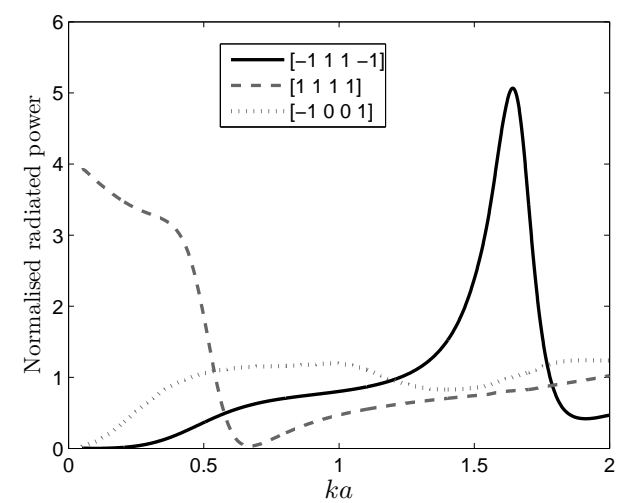

Figure 14. Eigenvalues of heave damping matrix, normalised by damping for an isolated cylinder.

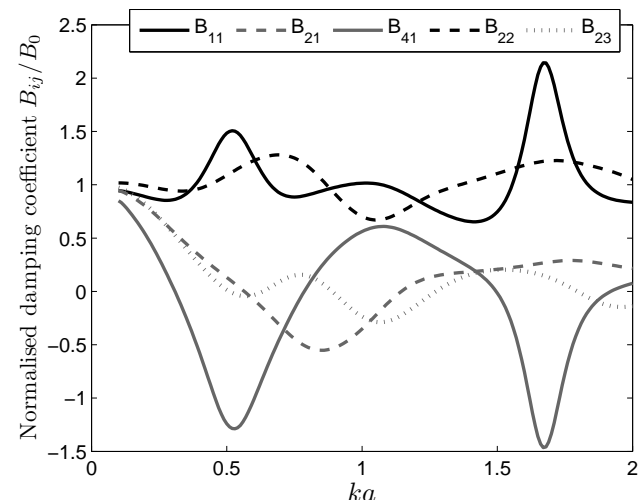

Figure 15. Individual elements of surge damping matrix, with each body restricted to $x^{\prime}$-surge. 


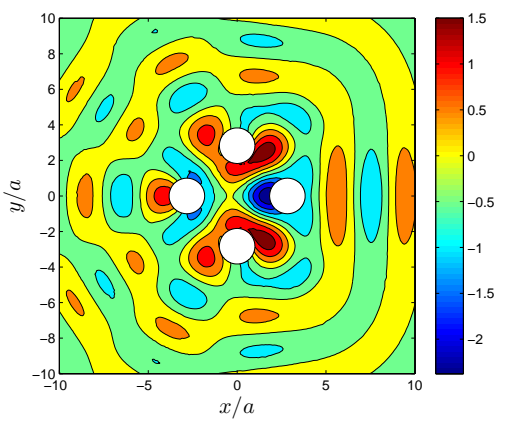

(a)

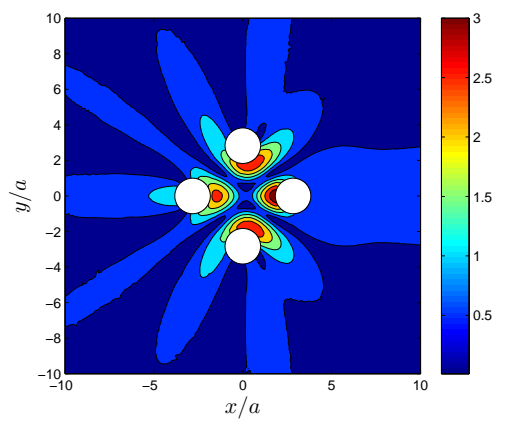

(b)

Figure 16. (a) Real part and (b) amplitude modulus of free surface elevations for scattered wave due to wave of unit amplitude incident on fixed array at $k a=1.66$ (incident wave subtracted).

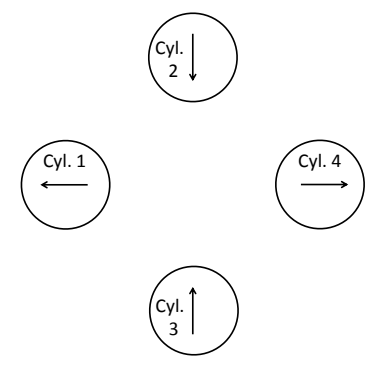

Figure 17. Pattern of horizontal exciting force determined by Porter and Evans [4] for sloshing near-trapped mode, with cylinders labelled as in Figure 1.

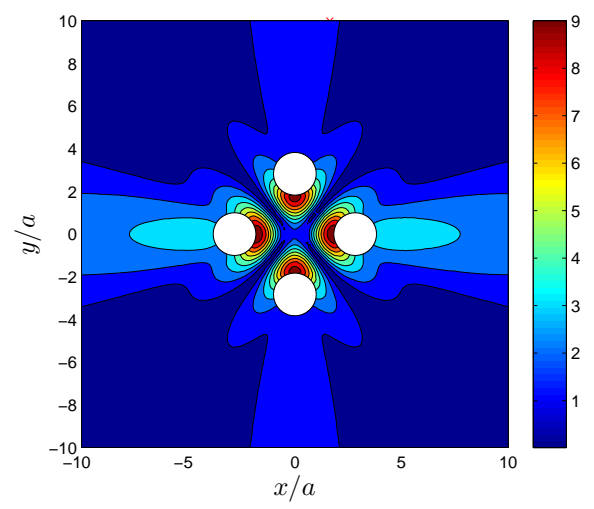

Figure 18. Radiation pattern for the $x^{\prime}$-surge $[1,0,0,-1]$ mode. The peaks around cylinders 1 and 4 are $\pi$ out of phase with those around cylinders 2 and 3 . 


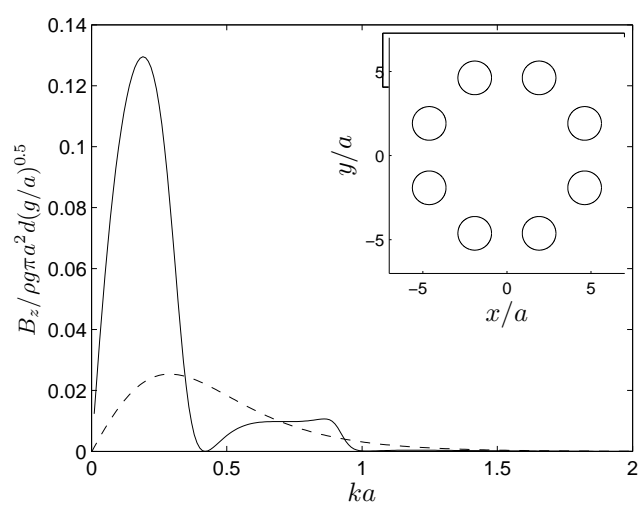

Figure 19. Heave damping coefficient for the 8 cylinder structure shown (in plan) in the inset, compared to the damping of 8 isolated cylinders (both draft $d=2 a$ ).

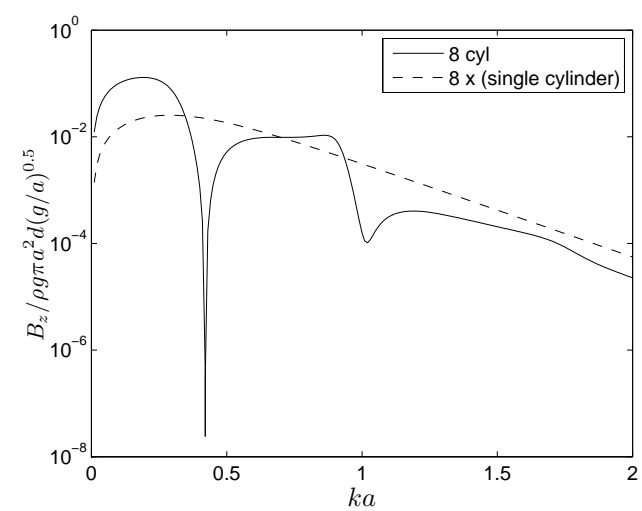

Figure 20. Heave damping coefficient for the 8 cylinder structure, $d=2 a$.

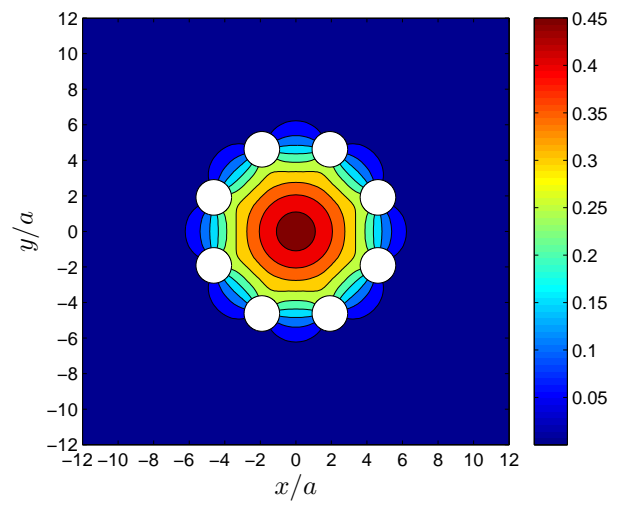

Figure 21. Modulus of free surface elevation around 8 cylinders (draft $d=2 a$ ) heaving as a single structure with unit amplitude, at wave free-frequency $k a=0.4216$. 


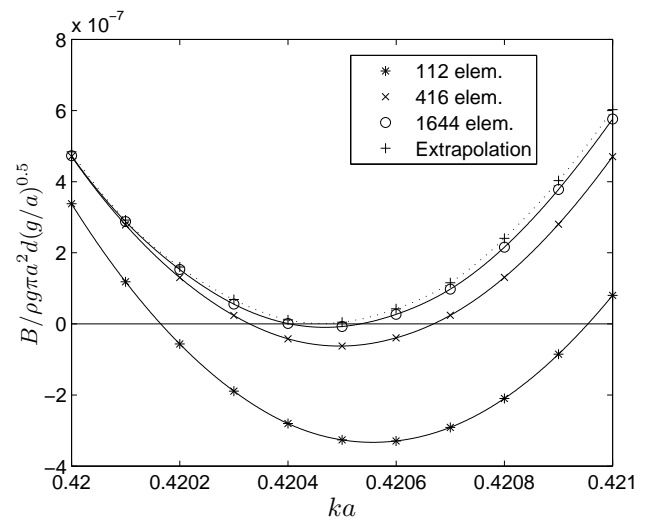

Figure 22. Heave damping coefficient for 8 cylinder structure, draft $d=2.068 a$, around the minimum damping frequency. Results for 3 meshes (labelled by number of elements per cylinder) and extrapolated results are shown.

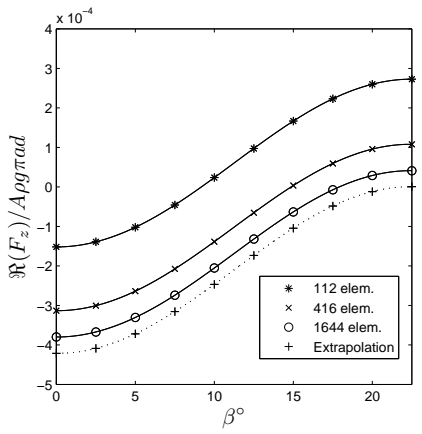

(a) Re

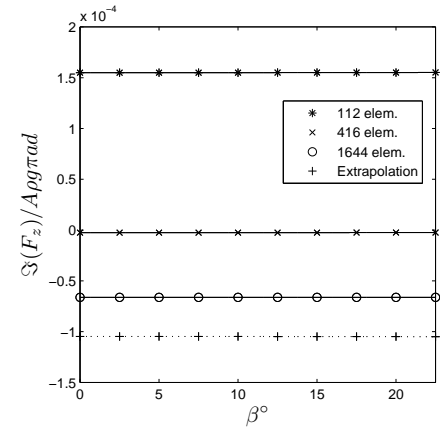

(b) Im

Figure 23. Variation of heave exciting force on 8 cylinder structure with incoming wave direction $\beta$, where structure has draft $2.068 a$ and incoming waves are of wavenumber $k a=0.4205$ and amplitude $A$. 


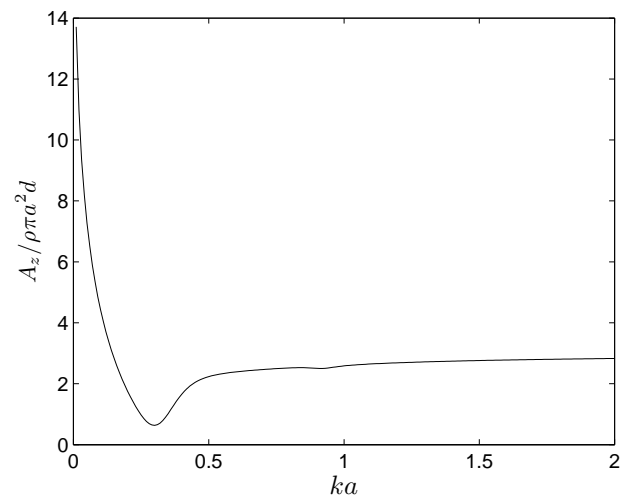

Figure 24. Heave added mass for 8 cylinder structure $(d=2 a)$.

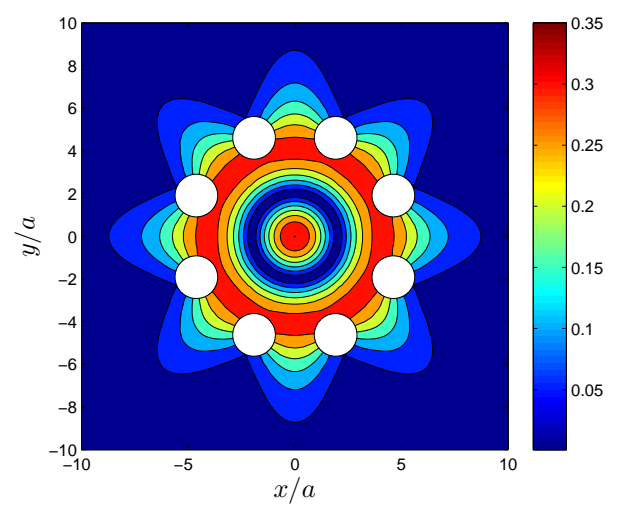

Figure 25. Modulus of free surface elevation around 8 cylinders (draft $d=2 a$ ) heaving as a single structure with unit amplitude, $k a=1.02$. Within the "moonpool" the central peak and surrounding ring are $\pi$ out of phase, in a standing wave-type pattern. 